\title{
Impending paradoxical embolism across the interatrial septum
}

\author{
Embolia paradoxal iminente através do septo interatrial
}

\author{
Nilson Lopez Ruiz
}

\section{To the Editor:}

Acute pulmonary embolism (PE) is considered a cardiovascular emergency because it can lead to acute life-threatening right ventricular failure. It has been reported that approximately 5\% of patients with acute PE present with right heart thrombus (RHT), ${ }^{(1-3)}$ which occasionally straddles the interatrial septum, posing a threat to the pulmonary arteries and systemic circulation.

A 63-year-old man presented to the Pablo Tobon Uribe Hospital, in Medellin, Colombia, complaining of dyspnea in the preceding two weeks. Physical examination revealed tachycardia, tachypnea, mild hypertension, jugular venous distention, abdominal distention, and edema in the lower limbs. However, cardiac and pulmonary findings were unremarkable. An electrocardiogram revealed only sinus tachycardia. The cardiac troponin 1 level was $0.2 \mathrm{ng} / \mathrm{mL}$ (normal, < $0.04 \mathrm{ng} / \mathrm{mL}$ ), and the N-terminal pro-brain natriuretic peptide level was $3,590 \mathrm{pg} / \mathrm{mL}$ (normal, < $125 \mathrm{pg} / \mathrm{mL}$ ). Subsequent helical CT scans of the chest confirmed PE by showing filling defects involving the main pulmonary artery and its primary branches (Figure 1). A venous ultrasound revealed left iliofemoral deep vein thrombosis. Intravenous heparin was started.

A bedside echocardiogram revealed a large, highly mobile, "snake-like" mass in the right atrium, attached to the interatrial septum (Figure 2) and penetrating into the left atrium (Video $1^{1}$ ). That image was also evident on a second review of the helical CT scans. The right ventricle (RV) was dilated (basal diameter, $4.5 \mathrm{~cm}$ ), hypokinetic (decreased global longitudinal strain; Figure 2), and pressure overloaded (systolic RV pressure, $45 \mathrm{mmHg}$ ).

Despite the RHT and the findings of impending systemic embolism, the cardiovascular surgeon refused to perform surgical thrombectomy because of the simultaneous, heavy clot burden on the pulmonary arteries. In addition, the size of the thrombus at the left atrium raised concerns regarding the risk of systemic embolism, which prevented us from performing thrombolysis. Hence, the patient was treated with anticoagulants only, and transesophageal echocardiography was deemed futile. Further diagnostic work-up confirmed malignant ascites, and the final diagnosis was adenocarcinoma of unknown primary origin. The clinical evolution was satisfactory. Because of heparin-induced thrombocytopenia type 1 (his platelet count fell down from 590,000 at admission to 102,000 after 8 days of heparin use), he was discharged on fondaparinux. However, the patient was readmitted several times for the palliative treatment of ascites. There was no clinical evidence of cardioembolic events until his death, which occurred four months later.

Currently, only the presence of RV strain on echocardiography and elevated biomarkers are recommended for assessing the need of thrombolysis in the setting of submassive PE. ${ }^{(4)}$ In rare cases, echocardiography can reveal RHT. In the International Cooperative Pulmonary Embolism Registry, 1,135 patients with PE were assessed by echocardiography, and 42 (4\%) were found to have RHT.(1) Likewise, two single-center studies, each including more than 300 patients, reported echocardiographic evidence of RHT in approximately 5\% of the patients. ${ }^{(2,3)}$ The appearance of RHT might suggest the etiology of the clot. ${ }^{(5)}$ Type A thrombi are tubular-shaped, highly mobile, and believed to represent peripheral venous clots temporarily lodged in the right heart. Type B thrombi resemble left heart thrombi, are less mobile, and appear to be associated with underlying cardiac abnormalities. Finally, type C thrombi are rare, are similar to myxomas in their appearance, and are highly mobile. The appearance of the thrombi has been associated with short-term prognosis. ${ }^{(5)}$ Not only is early mortality ( $<8$ days) higher for patients with type A than for those with type C or B (42\%,

1 Video available in the online version of the Brazilian Journal of Pulmonology

(http://www.jornaldepneumologia.com.br/portugues/artigo_detalhes.asp?id=1994). 
$14 \%$, and $4 \%$, respectively), but also the risk of pulmonary embolism is higher for those with type A than for those with type C or B (79\%, $62 \%$, and $38 \%$, respectively). However, the final outcome depends on the total clot burden and on RV myocardial performance rather than on the intracardiac thrombus alone. In addition, after hospital discharge, the prognosis appears to depend largely on the underlying disease (sepsis, tumor, heart disease, etc.) and not on the thrombus itself. ${ }^{(5)}$ When associated with cardiac septum defects, RHT poses a risk of paradoxical,

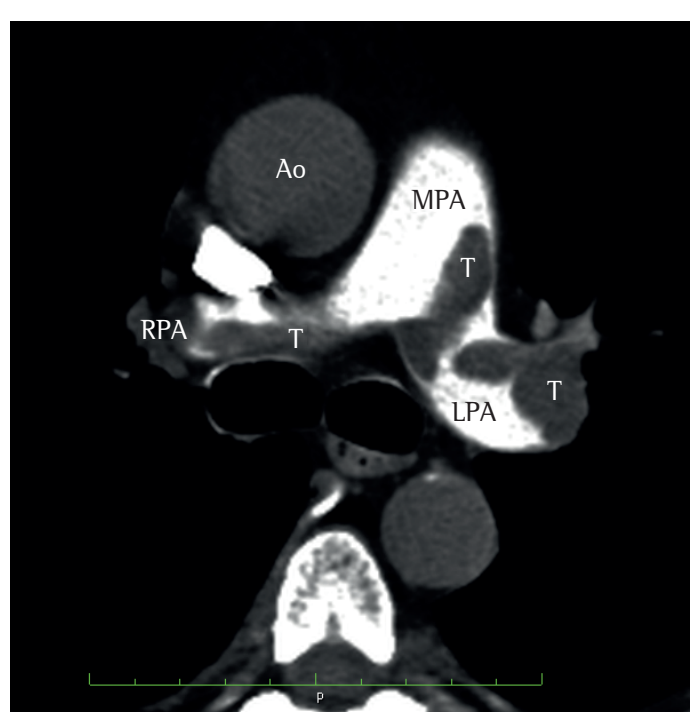

Figure 1 - CT scan of the chest showing various intraluminal filling defects-thrombi (T)-involving the main pulmonary artery (MPA), the left pulmonary artery (LPA), and the right branches of the right pulmonary artery (RPA). Ao: aorta. systemic embolism. Mostly, the thrombi pass through an interatrial septum defect or patent foramen ovale (PFO), from the right to the left cardiac chambers. A PFO increases the relative risk $(R R)$ of death $(R R=2.4)$, ischemic stroke $(R R=5.9)$, peripheral arterial embolism $(R R=15)$, and complicated hospital course $(R R=5.2){ }^{(4)}$ Recent guidelines recommend screening for PFO in patients with PE, ${ }^{(4)}$ although whether and when surgery is indicated remains unclear.

Finally, in the presence of massive or submassive PE, the treatment of PE in patients with impending systemic embolism remains controversial. According to a statement from the American Heart Association, ${ }^{(4)}$ surgical thrombectomy (particularly in patients with an intracardiac thrombus) might reduce the incidence of stroke, whereas thrombolysis might be associated with higher mortality. A recent systematic review ${ }^{(6)}$ supported that recommendation by showing that the incidence of the composite endpoint of 30-day mortality or systemic embolism during treatment was lower among patients undergoing surgical thrombectomy than among those treated with anticoagulants alone (OR $=0.26 ; 95 \% \mathrm{Cl}, 0.11-0.60 ; \mathrm{p}<0.001)$. That incidence was not significantly lower in those undergoing thrombolysis $(\mathrm{OR}=0.90 ; 95 \% \mathrm{Cl}, 0.28$ 2.86; $p=0.86)$. However, these recommendations might not be appropriate for cancer patients, in whom the risk of venous thromboembolism is higher and more conservative treatment would be advised, given that anticoagulation might suffice without producing any clinically significant thromboembolic complications.

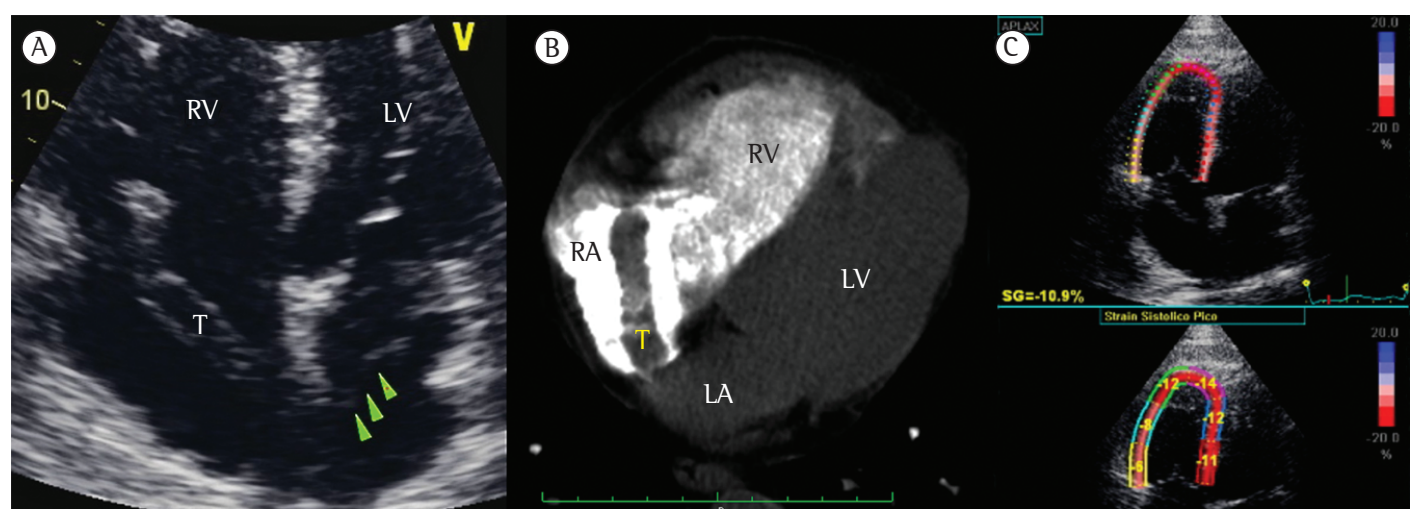

Figure 2 - In A, echocardiogram showing a large thrombus $(\mathrm{T})$ in the right atrium crossing the interatrial septum into the left atrium (arrowheads). In B, detail of a CT scan of the chest revealing a thrombus (T) in the right atrium (RA). In C, right ventricle (RV) dysfunction, as evidenced by decreased global longitudinal strain. LV: left ventricle; and LA: left atrium. 


\section{Nilson Lopez Ruiz} Attending Cardiologist, Unidad de Cardiologia, Hospital Pablo Tobon Uribe, Medellin, Colombia

\section{References}

1. Torbicki A, Galié N, Covezzoli A, Rossi E, De Rosa M, Goldhaber SZ, et al. Right heart thrombi in pulmonary embolism: results from the International Cooperative Pulmonary Embolism Registry. J Am Coll Cardiol. 2003;41(12):2245-51.

2. Pierre-Justin G, Pierard LA. Management of mobile right heart thrombi: a prospective series. Int $\mathrm{J}$ Cardiol. 2005;99(3):381-8.
3. Ferrari E, Benhamou M, Berthier F, Baudouy M. Mobile thrombi of the right heart in pulmonary embolism: delayed disappearance after thrombolytic treatment. Chest. 2005;127(3):1051-3.

4. Jaff MR, McMurtry MS, Archer SL, Cushman M, Goldenberg $\mathrm{N}$, Goldhaber SZ, et al. Management of massive and submassive pulmonary embolism, iliofemoral deep vein thrombosis, and chronic thromboembolic pulmonary hypertension: a scientific statement from the American Heart Association. Circulation. 2011;123(16):1788-830. Erratum in: Circulation. 2012;125(11):e495. Circulation. 2012;126(7):e104.

5. The European Cooperative Study on the clinical significance of right heart thrombi. European Working Group on Echocardiography. Eur Heart J. 1989;10(12):1046-59. 6. Myers PO, Bounameaux H, Panos A, Lerch R, Kalangos A. Impending paradoxical embolism: systematic review of prognostic factors and treatment. Chest. 2010;137(1):164-70. 